BMJ Open Sport \& Exercise Medicine

\section{Myocardial remodelling and tissue characterisation by cardiovascular magnetic resonance (CMR) in endurance athletes}

To cite: Pujadas S, Doñate M, Li C-H, et al. Myocardial remodelling and tissue characterisation by cardiovascular magnetic resonance (CMR) in endurance athletes. BMJ Open Sport \& Exercise Medicine 2018;4:e000422. doi:10.1136/ bmjsem-2018-000422

Accepted 3 October 2018
Check for updates

\section{(c) Author(s) (or their} employer(s)) 2018. Re-use permitted under CC BY-NC. No commercial re-use. See rights and permissions. Published by BMJ.

${ }^{1}$ Cardiac Imaging Unit, Cardiology Department, Hospital de la Santa Creu i Sant Pau, Autonomous University of Barcelona, Barcelona, Spain ${ }^{2}$ Radiology Department, Clínica Creu Blanca, Barcelona, Spain

Correspondence to Dr Sandra Pujadas; sandrapujadas@gmail.com

\section{ABSTRACT}

There is still some controversy about the benignity of structural changes observed in athlete's heart, especially regarding the observation of increased biomarkers and the presence of myocardial fibrosis (MF).

Aim Our purpose was to evaluate by cardiovascular magnetic resonance (CMR) the presence of diffuse as well as focal MF in a series of high-performance veteran endurance athletes.

Methods Thirty-four veteran healthy male endurance athletes, still being in regular training, with more than 10 years of training underwent a CMR. A cardiopulmonary exercise test was also performed to assess their maximal physical performance. The control group consisted in 12 non-trained normal individuals.

Results We found an increase in both, right and left ventricular (LV) volumes in the athlete's group when compared with controls. There was no increase in indexed LV myocardial mass despite of a significantly increased maximal myocardial wall thickness in comparison to controls. Native T1 values and extracellular volume (ECV) were normal in all cases. We did not find differences in native T1 values and ECV between both groups. In three athletes $(9 \%)$, non-ischaemic late gadolinium enhancement (LGE) was observed. We did not find a correlation between total training volume and presence of LGE or with the ECV value.

Conclusions Our results show that the majority of veteran endurance athletes present with myocardial remodelling without MF as a physiological adaptive phenomenon. In the only three athletes with focal MF, the LGE pattern observed suggests an intercurrent event not related with the remodelling phenomenon.

\section{INTRODUCTION}

Structural changes observed in athlete's heart such as increased left ventricular (LV) volume, increased LV wall thickness and mass have been generally considered as benign consequences of long-term physical training. However, several studies have demonstrated that acute high-intensity endurance exercise such as marathon or ultramarathon courses

\section{What are the new findings?}

Myocardial changes induced by long-term endurance exercise do not result in pathological hypertrophy or myocardial fibrosis.

How might it impact on clinical practice in the near future?

These findings allow a better knowledge of athlete's heart in veteran endurance athletes. Cardiovascula magnetic resonance results show that structural changes observed in these population correspond to a physiological adaptative phenomenon.

is associated with transient decrease in right ventricular systolic function. ${ }^{1-3}$ Increases in cardiac biomarkers of myocardial injury, such as troponine, have been reported to occur immediately after performing these activities. ${ }^{45}$ Recently, it has been described in an experimental study an association between long-term vigorous endurance exercise, arrhythmias and myocardial fibrosis (MF). ${ }^{6}$

Cardiac magnetic resonance (CMR) imaging allows the detection of replacement MF by means of the technique of late gadolinium enhancement (LGE). More recently, the estimation of the longitudinal magnetic relaxation time $\mathrm{T} 1$ of the myocardial tissue, from which a calculation of the myocardial extracellular volume (ECV) may be derived, this being a reliable measure of diffuse interstitial fibrosis.

Although some CMR studies have already shown the presence of focal MF in athletes, ${ }^{78}$ it is not known at present whether high-intensity endurance exercise might result in diffuse MF. 
Our purpose was to evaluate by CMR the presence of diffuse as well as focal MF in a series of high-performance veteran endurance athletes.

\section{METHODS}

\section{Study population}

Thirty-four veteran healthy male (age $48.17 \pm 7.48$ years) endurance athletes (runners), still being in regular training, with more than 10 years of training and with marathon times below 3 hours and $15 \mathrm{~min}$, were invited to undergo a CMR. A cardiopulmonary exercise test was also performed in all individuals to assess their maximal physical performance. The control group consisted of 12 untrained healthy males pair-matched for age and body surface area. Exclusion criteria included any history of cardiopulmonary disease and standard contraindications for CMR imaging.

The study was conducted in accordance with the standards set by the 'Declaration of Helsinki' and approved by the ethics committee of our Institution.

\section{Cardiovascular magnetic resonance}

All CMR studies were performed with a $1.5 \mathrm{~T}$ magnet (Avanto Fit, Siemens; Erlangen, Germany). LV and RV mass and volumes were obtained from steady-state free precession cine imaging covering the entire LV in the short-axis: retrospective ECG-gated SSFP cine sequence $(\mathrm{TR}=74 \mathrm{~ms}, \mathrm{TE}=1.26 \mathrm{~ms}$, slice thickness $=8 \mathrm{~mm}$, no gap, voxel size $1.3 \times 1.3 \times 8 \mathrm{~mm})$. Cine images were analysed by means of dedicated software (QMass 7.1 Medis, Leiden, The Netherlands).

Mid LV short-axis native (precontrast) T1 maps were obtained using a previously described MOLLI sequence. ${ }^{9}$

A single bolus of $0.2 \mathrm{mmol} / \mathrm{kg}$ of gadobutrol (Gadovist, Bayer Lab. Barcelona, Spain) was administered. A 3-dimensional inversion recovery segmented gradient echo sequence (spatial resolution $1.3 \times 1.3 \times 8 \mathrm{~mm}$ ) was acquired $8-10 \mathrm{~min}$ after contrast administration to assess LGE. This sequence was used in multiple shortaxis planes using the same orientation as the cine MR images.

Post-contrast T1 maps were acquired using the same MOLLI scheme 15 min after contrast administration.

Focal MF was considered to be present when an intramyocardial region with hyperintense signal $(>5$ $\mathrm{SD}$ values of the signal intensity at a remote area) was detected in the LGE images.

Diffuse MF was assessed using T1 mapping sequences by means of calculation of the ECV according to the equation: $\mathrm{ECV}=\Delta 1 / T 1_{\text {myocardium }} / \Delta 1 / T 1_{\text {blood }}$ (1-haematocrit). ${ }^{10}$ Haematocrit was obtained at the moment of the CMR study.

\section{Cardiopulmonary exercise test}

Exercise test with gas consumption was performed in all athletes on an Ergo-Run premium 8 ergometer (Daum-electronic, Fürth, Germany) using a previously validated $^{11}$ progressive protocol. After a warm-up of 3 $\min$ at $6 \mathrm{~km} /$ hour with a fixed ramp of $3 \%$, the velocity was increased $1 \mathrm{~km} /$ hour every minute until exhaustion. A simultaneous breath by breath gas exchange device (Power-Cube, Ganshorn, Neuenkirchen, Germany) was used to measure: oxygen consumption $\left(\mathrm{VO}_{2}\right)$ (in $\mathrm{L} / \mathrm{min}$ and $\mathrm{ml} / \mathrm{kg} / \mathrm{min}$ ); carbon dioxide production $\left(\mathrm{VCO}_{2}\right)$ (in $\left.\mathrm{L} / \mathrm{min}\right)$; respiratory quotient (RER); anaerobic threshold (UAn) based on heart rate, percentage of $\mathrm{VO}_{2}$ achieved at the UAn $\left(\% \mathrm{VO}_{2}\right)$ and ventilation/ minute (VE) (in L/min). Signals from a 12 lead ECG were displayed continuously using a Cardiovit AT-104 system (Schiller España, Madrid, Spain). Blood pressure was measured at 3 min intervals during exercise and for the first $3 \mathrm{~min}$ after exercise, using a manual sphygmomanometer. Criteria for termination of exercise test were to yield physical exhaustion or a plateau in $\mathrm{VO}_{2}$. Maximal cardiopulmonary exercise test was defined by a plateau in $\mathrm{VO}_{2}$ and/or achievement of a peak RER of $\geq 1.10$

\section{Statistical analysis}

Continuous variables are presented as mean $\pm \mathrm{SD}$ when normally distributed and median $\pm \mathrm{IQR}$ when not. Normal distribution of data was assessed by using Shappiro-Wilk test.

To analyse the differences between pairwise matched athletes and control subjects a Student's t-test was used. Wilcoxon signed-rank test was performed for non-normally distributed variables. A multivariate-logistic regression model was used to control for confounding baseline variables. All $\mathrm{p}<0.05$, 2-tailed approach, and power of $80 \%$ were used in all tests.

\section{RESULTS}

Patient demographics and functional measures are summarised in table $1 . \mathrm{VO}_{2}$ values shown in the athlete's group were in the range of well highly trained subjects according to reference values. ${ }^{11}$

CMR results are shown in table 2. We found an increase in both right and LV volumes in the athlete's group when compared with controls. Left and right ejection fractions were preserved in all patients. There was no increase in indexed LV myocardial mass despite of a significantly increased maximal myocardial wall thickness in comparison to controls.

Native T1 values and ECV were normal in all cases. We found significant differences between athletes and control subjects in the univariate analysis. When corrected by the haematocrit in the multivariate analysis, we did not find differences in native $\mathrm{T} 1$ values and ECV between both groups (table 2).

In three athletes (9\%), non-ischaemic LGE pattern was noted: mesocardial in septal-apical wall, subepicardial in the inferior apical wall and mesocardial in the lateral wall (figure 1). 
Table 1 Demographic and functional characteristics of endurance athletes and control subjects

\begin{tabular}{lccl}
\hline & Athletes $(\mathbf{n}=\mathbf{3 4})$ & Controls $(\mathbf{n}=\mathbf{1 2})$ & P values \\
\hline Age (years) & $48.18 \pm 7.48$ & $42.36 \pm 13.43$ & n.s \\
BSA $\left(\mathrm{m}^{2}\right)$ & $1.80 \pm 0.11$ & $1.89 \pm 0.14$ & $\mathrm{n} . \mathrm{s}$ \\
Training volume (hours/week) & $9.38 \pm 3.52$ & $3.08 \pm 1.52$ & $<0.05$ \\
Training history (years) & $28.06 \pm 10.84$ & $9.38 \pm 3.02$ & $<0.05$ \\
Rest heart rate (bpm) & $56.03 \pm 8.87$ & $70.33 \pm 15.42$ & $<0.05$ \\
$\mathrm{VO}_{2}$ peak $(\mathrm{mL} / \mathrm{min} / \mathrm{kg})$ & $60 \pm 6.53$ & & \\
Peak heart rate (bpm) & $176.27 \pm 10.32$ & & \\
RER max & $1.14 \pm 0.07$ & & \\
\hline
\end{tabular}

Data are presented as mean value $\pm \mathrm{SD}$. $\mathrm{VO}_{2}$ peak, maximal oxygen uptake.

BSA, body surface area; RER max, maximal respiratory exchange ratio.

We did not find a correlation between total training volume and presence of LGE nor with the ECV value.

\section{DISCUSSION}

In this study, we have examined a cohort of veteran endurance athletes and a paired untrained control group to evaluate the consequences of long time practice of intense endurance exercise on myocardial structure to find that:
1. Cardiac remodelling in endurance athletes is characterised by a balanced increase in left and right ventricular volumes and in maximal LV wall thickness, despite of normal indexed myocardial mass.

2. This remodelling process does not involve an expansion of the interstitial space by MF, as proven by the normal values of the ECV, suggesting a physiological adaptive phenomenon in these highly trained individuals.

\begin{tabular}{|c|c|c|c|c|}
\hline & Athletes $(m e a n \pm S D)$ & Controls (mean $\pm S D$ ) & $P$ values & \\
\hline LVEDV (mL) & $193.65 \pm 31.67$ & $155.92 \pm 26.30$ & $<0.001$ & \\
\hline LVEDV index $\left(\mathrm{mL} / \mathrm{m}^{2}\right)$ & $107.53 \pm 15.94$ & $81.33 \pm 10.71$ & $<0.001$ & \\
\hline LVESV (mL) & $72.47 \pm 15.98$ & $57.75 \pm 13.96$ & $<0.001$ & \\
\hline LVESV index $\left(\mathrm{mL} / \mathrm{m}^{2}\right)$ & $40.29 \pm 8.59$ & $30.08 \pm 6.32$ & $<0.001$ & \\
\hline LVEF (\%) & $62.53 \pm 4.89$ & $63 \pm 5.15$ & 0.779 & \\
\hline RVEDV (mL) & $204.44 \pm 37.50$ & $165.58 \pm 31.90$ & 0.003 & \\
\hline RVEDV index $\left(\mathrm{mL} / \mathrm{m}^{2}\right)$ & $113.59 \pm 19.83$ & $86.42 \pm 13.57$ & $<0.001$ & \\
\hline RVESV (mL) & $76.12 \pm 20.69$ & $64.67 \pm 17.15$ & $0.086^{\star}$ & \\
\hline RVESV index $\left(\mathrm{mL} / \mathrm{m}^{2}\right)$ & $42.38 \pm 11.61$ & $33.83 \pm 7.85$ & $0.023^{*}$ & \\
\hline RVEF (\%) & $62.91 \pm 6.24$ & $61.5 \pm 5.27$ & 0.488 & \\
\hline Ratio RV-LV & $0.96 \pm 0.10$ & $0.96 \pm 0.14$ & 0.968 & \\
\hline LVM (g) & $124.59 \pm 22.13$ & $124.54 \pm 32.23$ & 0.996 & \\
\hline LVM index $\left(\mathrm{g} / \mathrm{m}^{2}\right)$ & $69 \pm 11.05$ & $65.25 \pm 15.73$ & 0.372 & \\
\hline Max wall thickness (mm) & $11 \pm 1.33$ & $8.50 \pm 2.65$ & $<0.001^{*}$ & \\
\hline LA area $\left(\mathrm{cm}^{2}\right)$ & $28.03 \pm 3.71$ & $24.67 \pm 4.16$ & 0.013 & \\
\hline \multicolumn{5}{|l|}{ T1 mapping } \\
\hline Native T1 septal (ms) & $943.59 \pm 52.58$ & $984.13 \pm 36.82$ & $0.006^{*}$ & NS \\
\hline Native T1Lat (ms) & $925.3 \pm 45.90$ & $960.25 \pm 29.77$ & $0.029^{\star}$ & NS \\
\hline ECV septal & $0.25 \pm 0.02$ & $0.22 \pm 0.02$ & $<0.001$ & NS \\
\hline ECVLat & $0.24 \pm 0.04$ & $0.17 \pm 0.08$ & 0.006 & NS \\
\hline Haematocrit (\%) & $0.42 \pm 0.02$ & $0.46 \pm 0.04$ & $0.001^{*}$ & $S$ \\
\hline $\mathrm{DE}^{*}$ & $3(9 \%)$ & 0 & & \\
\hline
\end{tabular}

CMR, cardiovascular magnetic resonance; DE, delayed-enhancement; ECV, extracellular volume; LA, left atrial; LVEDV, left ventricular end-diastolic volume; LVEF, left ventricular ejection fraction; LVM, left ventricular mass; RVEDV, right ventricular end-diastolic volume; RVEF, right ventricular ejection fraction. 


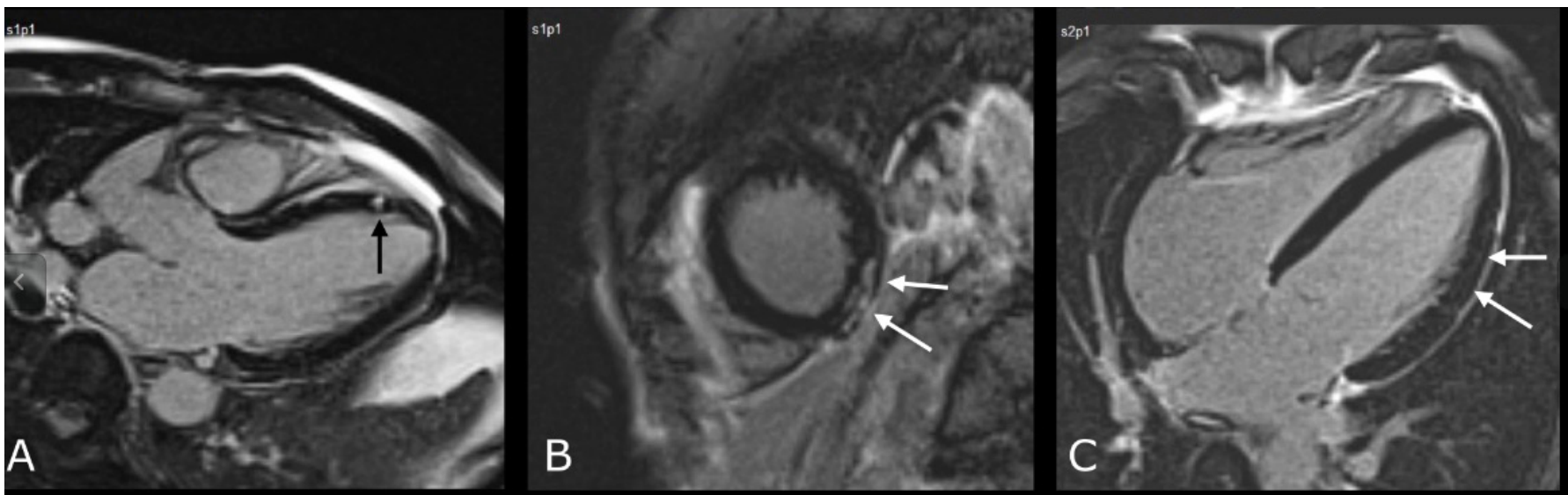

Figure 1 LGE images of the three athletes showing MF. (A) A 55-year-old athlete who has been training 10 hours/week in the last 28 years. Mesocardial LGE in the apical-septal wall is shown in a 3-chamber view image. (B) A 51-year-old athlete training 7 hours/week in the last 30 years. The short-axis view shows subepicardial LGE in the inferior apical wall. (C) A 55-year-old athlete training 8 hours/week in the last 30 years. Mild intramyocardial LGE is the lateral wall is shown in the 4-chamber view. LGE, late gadolinium enhancement; MF, myocardial fibrosis.

3. In $9 \%$ of athletes, however, focal MF was observed, which could be due to an intercurrent phenomenon of uncertain origin.

\section{Morphological and functional parameters}

Cardiac adaptation in veteran, high-performance endurance athletes is characterised by balanced increase of LV and $\mathrm{RV}$ volumes, increased $\mathrm{LV}$ wall thickness and myocardial mass, ${ }^{12}{ }^{13}$ leading to what is known as the athlete's heart. Accordingly, our cohort showed significantly enlarged left and right ventricles compared to control subjects, with no significant differences in the LVEDVto-RVEDV ratio. Also, athletes showed an increase in absolute wall thickness compared to control subjects. However, in accordance with previous reports, ${ }^{14}$ values of indexed LV mass are within normal ranges. The present study also proves the preservation of LV and RV ejection fraction in lifelong endurance veteran athletes.

\section{Focal myocardial fibrosis}

LGE was observed in three (9\%) athletes, all of them showing a non-ischaemic pattern (figure 1A-C). Furthermore, in two of them, delayed-enhancement patterns were highly suggestive of a previous episode of clinically undetected myocarditis (figure $1 \mathrm{~B}, \mathrm{C}$ ).

There is still some controversy regarding on whether repeated extreme endurance exercise might result in MF. Benito et at exercised rats on a treadmill and observed diffuse fibrosis in the atria and right ventricle which resulted in ventricular arrhythmia. CMR studies have also reported an apparently high incidence of focal MF in veteran athletes: Breuckmann et al examined 102 healthy veteran male marathon runners and reported an unexpectedly high prevalence of LGE (12\%), although it was not significantly different from that of age-matched control participants $(4 \%, \mathrm{p}=0.07)$. Another study ${ }^{15}$ found focal MF by LGE in 6 of 12 elite veteran athletes but not in 20 age-matched controls or in 17 young endurance athletes. The presence of LGE in veteran athletes was significantly associated with the training volume, supporting a link between life-long endurance exercise and MF.

More recently, La Gerche $e t a l^{16}$ showed LGE in 5 of 40 $(13 \%)$ athletes studied which was confined to the interventricular septum in all cases.

In two of the three athletes, we found a LGE pattern highly suggestive of a previous myocarditis as the potential cause. It would be reasonable to believe that continued exercise training may accelerate myocardial damage and MF during silent myocarditis. ${ }^{17}$

MF in the RV insertion point has been reported in up to $19 \%$ of athletes in the literature,$^{18}$ and it is an important topic of debate. It is speculated that the RV distention during exercise may result in chronic structural changes of the myocardial architecture, mainly in the septal points of insertion of the right ventricular wall. In our study, we found mild LGE in the inferior septal RV insertion in $23 \%$ of athletes. However, we did not consider this finding as true reactive MF. In fact, this finding was also observed in $36 \%$ of controls (figure 2). We believe that, as previously reported, ${ }^{17} 19$ plexiform fibrosis is a normal feature of the insertion-region anatomy that may result in contrast pooling within this area.

\section{DIFFUSE MYOCARDIAL FIBROSIS}

Importantly, we did not find significant differences in ECV in athletes compared with control group. Extracellular expansion leads to higher ECV measurements. Thus, our findings support that cardiac remodelling in veteran athletes is a physiological adaptive phenomenon.

Previously, McDiarmid et $a l^{20}$ showed in young athletes that the increase in LV mass is due to increased cellular mass which, in turn, results in a relative decrease in ECV compared with controls. These findings also support the hypothesis of a physiological adaptation instead of pathological hypertrophy with associated interstitial fibrosis. ${ }^{21}$ 


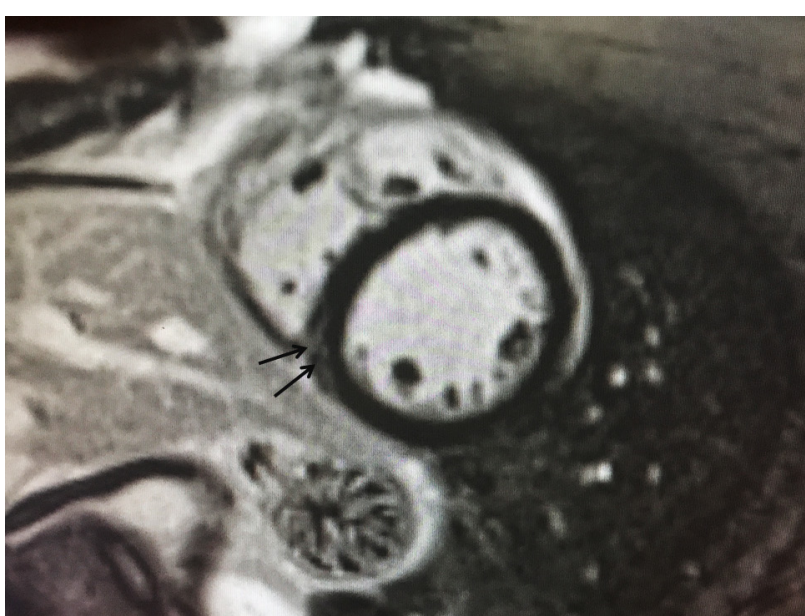

Figure 2 A 40-year-old man with a training history of 2 hours/week during 6 years (Control group). A mid-ventricular short-axis view shows mild LGE in the inferior RV-LV junction.

However, in the present study, we did not find significant differences in ECV between athletes and controls. This discrepancy may be explained by differences in athlete population between McDiarmid's study and ours. Our study comprised veteran endurance athletes (mean age: $47.18 \pm 7.48)$, much older than the study subjects from their study (31.7 \pm 7.7 years), and our results are according to a previous study by Mordi et $a t^{22}$ also with veteran endurance athletes in which they did not find a decrease in ECV in athletes compared with sedentary controls. More studies will be required to confirm whether there are significant differences in ECV between young and veteran athletes.

In our population of veteran runners with more than 10 years of intense training, we did not find a correlation between total training volume and MF of any kind and neither between ECV and maximal wall thickness nor LV mass. Thus, according to our results, running at high doses and intensities does not seem to result in developing interstitial fibrosis. However, more studies are needed including young and veteran athletes and from different sports.

\section{LIMITATIONS}

Only 46 subjects were enrolled in this study, which may be a limitation when comparing structural cardiac changes between the two groups, particularly when assessing myocardial LGE patterns.

A cardiopulmonary exercise test to the control group was not performed, as the only purpose of this test was to show the excellent athletic performance of the study group. The present study is only referred to Caucasian male veteran athletes as we did not study black, young or female athletes.

\section{CONCLUSION}

Our results show that the majority of veteran endurance athletes present with myocardial remodelling without MF as a physiological adaptive phenomenon. Furthermore, in the only three athletes with focal MF, the LGE pattern observed suggests an intercurrent event not related with their modelling phenomenon.

Acknowledgements We would like to thank Cors Units Foundation for their disinterested support of this project.

Contributors Conception and study design: SP, FC and RS-G. Data collection: MD, AC and XA. Data analysis: C-HL. Interpretation of data and results: SP, FC and SM. Writing the draft: SP and SM. Reviewing manuscript: FC and GP-L. All authors read and approved the final manuscript.

Competing interests None declared.

Patient consent Obtained.

Ethics approval The study was approved the ethics committee of Institut de Recerca of Hospital de Sant Pau: IIBSP-ATL-2017-91

Provenance and peer review Not commissioned; externally peer reviewed.

Data sharing statement All data analysed during this study are included in this published article.

Open access This is an open access article distributed in accordance with the Creative Commons Attribution Non Commercial (CC BY-NC 4.0) license, which permits others to distribute, remix, adapt, build upon this work non-commercially, and license their derivative works on different terms, provided the original work is properly cited, appropriate credit is given, any changes made indicated, and the use is non-commercial. See: http://creativecommons.org/licenses/by-nc/4.0/

\section{REFERENCES}

1. Claessen G, Claus P, Delcroix M, et al. Interaction between respiration and right versus left ventricular volumes at rest and during exercise: a real-time cardiac magnetic resonance study. Am J Physiol Heart Circ Physiol 2014;306:H816-24.

2. La Gerche A, Claessen G, Dymarkowski S, et al. Exerciseinduced right ventricular dysfunction is associated with ventricular arrhythmias in endurance athletes. Eur Heart J 2015;36:1998-2010.

3. Carrió I, Serra-Grima R, Berná L, LI B, et al. Transient alterations in cardiac performance after a six-hour race. Am J Cardiol 1990;65:1471-4.

4. Shave R, Baggish A, George K, et al. Exercise-induced cardiac troponin elevation: evidence, mechanisms, and implications. J Am Coll Cardiol 2010;56:169-76.

5. Shave R, George K, Gaze D. The influence of exercise upon cardiac biomarkers: a practical guide for clinicians and scientists. Curr Med Chem 2007;14:1427-36.

6. Benito B, Gay-Jordi G, Serrano-Mollar A, et al. Cardiac arrhythmogenic remodeling in a rat model of long-term intensive exercise training. Circulation 2011;123:13-22.

7. Breuckmann F, Möhlenkamp S, Nassenstein K, et al. Myocardial late gadolinium enhancement: prevalence, pattern, and prognostic relevance in marathon runners. Radiology 2009;251:50-7.

8. Möhlenkamp S, Lehmann N, Breuckmann F, et al. Running: the risk of coronary events : prevalence and prognostic relevance of coronary atherosclerosis in marathon runners. Eur Heart $J$ 2008;29:1903-10.

9. Taylor AJ, Salerno M, Dharmakumar R, et al. T1 Mapping: basic techniques and clinical applications. JACC Cardiovasc Imaging 2016;9:67-81.

10. Schelbert EB, Testa SM, Meier CG, et al. Myocardial extravascular extracellular volume fraction measurement by gadolinium cardiovascular magnetic resonance in humans: slow infusion versus bolus. J Cardiovasc Magn Reson 2011;13:16.

11. Balady GJ, Arena R, Sietsema K, et al. Clinician's Guide to cardiopulmonary exercise testing in adults: a scientific statement from the American Heart Association. Circulation 2010;122:191-225.

12. Pelliccia A, Culasso F, Di Paolo FM, et al. Physiologic left ventricular cavity dilatation in elite athletes. Ann Intern Med 1999;130:23-31.

13. Scharhag J, Schneider G, Urhausen A, et al. Athlete's heart: right and left ventricular mass and function in male endurance athletes and untrained individuals determined by magnetic resonance imaging. J Am Coll Cardiol 2002;40:1856-63.

14. Spirito P, Pelliccia A, Proschan MA, et al. Morphology of the "athlete's heart" assessed by echocardiography in 947 elite athletes representing 27 sports. Am J Cardiol 1994;74:802-6. 
15. Wilson M, O'Hanlon R, Prasad S, et al. Diverse patterns of myocardial fibrosis in lifelong, veteran endurance athletes. $\mathrm{J} \mathrm{Appl}$ Physiol 2011;110:1622-6.

16. La Gerche A, Burns AT, Mooney DJ, et al. Exercise-induced right ventricular dysfunction and structural remodelling in endurance athletes. Eur Heart J 2012;33:998-1006.

17. Cabinian AE, Kiel RJ, Smith F, et al. Modification of exerciseaggravated coxsackievirus B3 murine myocarditis by $T$ lymphocyte suppression in a inbred model. J Lab Clin Med;190:454-62.

18. van de Schoor FR, Aengevaeren VL, Hopman MT, et al. Myocardial fibrosis in athletes. Mayo Clin Proc 2016;91:1617-31.
19. Bradlow WM, Assomull R, Kilner PJ, et al. Understanding late gadolinium enhancement in pulmonary hypertension. Circ Cardiovasc Imaging 2010;3:501-3.

20. McDiarmid AK, Swoboda PP, Erhayiem B, et al. Athletic cardiac adaptation in males is a consequence of elevated myocyte mass. Circ Cardiovasc Imaging 2016;9:e003579.

21. Sado DM, Flett AS, Banypersad SM, et al. Cardiovascular magnetic resonance measurement of myocardial extracellular volume in health and disease. Heart 2012;98:1436-41.

22. Mordi I, Carrick D, Bezerra H, et al. T1 and T2 mapping for early diagnosis of dilated non-ischaemic cardiomyopathy in middle-aged patients and differentiation from normal physiological adaptation. Eur Heart J Cardiovasc Imaging 2016;17:797-803. 